\title{
Modified polylactide composites
}

\author{
Nataliya Semenyuk, Khrystyna Kysil, Galyna Dudok, Volodymyr Skorokhoda
}

Lviv Polytechnic National University, S. Bandery st., 12, Lviv, 79013, Ukraine, e-mail: nataliia.b.semeniuk@lpnu.ua

\begin{abstract}
The basic technological principles of obtaining modified polylactide materials in the films form and porous matrices for special application are investigated. Modification of polylactides with glycerol, polyvinylpyrrolidone, and heat treatment has been committed. The influence of synthesis conditions, composition composition on structure, morphology and properties of modified polylactide materials are revealed.
\end{abstract}

Keywords - polylactide, modified films, composites, porous polymer matrices, polyvinylpirrolidone.

Aggravation of the problems related to the plastic waste utilization has prompted to the searching and developing of technology to obtain biodegradable polymers which are able for decomposition in environment in a relatively short time. One of the most perspectives of this group of polymers is polylactide (PLA), which is biodegradable, biocompatible, thermoplastic polymer. It is capable to be processed into products from both melt and solution. PLA is applied in medicine, pharmacy, agriculture as well as in textile industry, packaging industry, paper lamination and food industry, etc. [1,2].

Polylactides modification can significantly expand the areas of its application also improve their properties $[3,4]$. Therefore, the research on the creation of modified PLA is relevant.

In the work the fundamentals of derivation technology of the modified polylactide materials with adjustable properties of the special purpose are developed.

The research was conducted in two directions: 1 - creation of modified PLA film materials for the packaging industry and paper lamination; 2- new porous modified PLA matrices development for application in medicine, in particular for osteoplasty.

To directly influence on the properties of PLA materials, its structure and morphology the polylactides were modified with glycerol and polyvinylpyrrolidone as well as by physical action, in particular, heat treatment.

At the first stage, the peculiarities of film formation on the basis of molified PLA by watering from the solution were researched. The given method made it possible to combine the process of film formation with subsequent modification of the properties of the initial polymers in the system. To improve the elasticity of the forming films the plasticizers, in particular glycerol, were added into the polymer solution. The selection of optimal solvent for the film formation by watering, namely chloroform was conducted. Concentration, temperature and time modes of the formation of modified PLA films are substantiated.

The influence of the solvent nature on viscosity characteristics of PLA solutions has been studied. Chloroform affects the crystallinity of films owing to conformational changes. Based on the conducted viscosity studies the molecular weight of PLA of the different brands, which are being applied for film formation, was determined.

The structure of films depends on the velocity and time of the solvent evaporation. The kinetics of solvent evaporation at the different temperature and time conditions has been studied. At a temperature of $50{ }^{\circ} \mathrm{C}$ there is a rapid evaporation of chloroform from the solution. Right away after 15 minutes from the beginning of evaporation the solvent almost does not remain. Evaporation of chloroform from PLA solutions and PLA blends with glycerol occurs at almost the same velocity.

By the SEM studies it is confirmed that the surface morphology of composite films with PLA is being determined via many factors, in particular, the nature of the polymer, the content and nature of the plasticizer, the 
temperature of the molding modes. Through the changing of mentioned factors, it is possible to influence on the structure of composites and their properties.

Infrared spectroscopic studies of PLA films, which are modified as well as heat-treated were performed that was confirmed an existence of absorption bands which are typical for PLA. The film's spectra based on PLA and glycerol blends confirm the presence of -OH group on the absorption spectrum.

According to thermal analysis, it was found that samples based on PLA and PLA with glycerol blends are characterized by high heat resistance and thermal resistance. The beginning of intensive mass loss of the samples caused by deep destructive and thermo-oxidative processes shifted to the diapason of high temperatures $\left(265^{\circ} \mathrm{C}\right.$ and above).

Influence of the conditions of film formation, glycerol amount, nature of PLA on physicalmechanical and elastic-deformation properties of modified PLA materials has been researched. The most strength films were formed applying the Luminy LX175 PLA.

An important modern direction of PLA composites usage is medicine. The features of porous matrices formation based on PLA in the presence of polyvinylpyrrolidone have been studied. The total porosity of the obtained composites is $70 \ldots 80 \%$, the total pore diameter is $280 \ldots 1000 \mu \mathrm{m}$. Since PLA composites do not satisfy the requirements concerning fungibactericidal properties, therefore in the given work it was modified with polyvinylpyrrolidone which can be used as a reducing agent during silver nanoparticles derivation in the composites [5-7].

A comparative analysis of the bactericidal and fungicidal properties of composites containing silver nanoparticles obtained by the PVP reduction reaction and non-silver-containing composites showed that composites containing silver nanoparticles block the growth of bacteria and fungi, demonstrating the fungibactericidal ability.

\section{References}

[1]. Muller, J., González-Martínez, Ch., Chiralt, A. (2017). Combination of Poly(lactic) Acid and Starch for Biodegradable Food Packaging. Materials, 10(8), 952-974. doi: 10.3390/ma10080952.

[2]. Malinconico, M., Vink, E., Cain, A. (2018). Industrial Applications of Poly(lactic acid). Cham: Springer International Publishing AG.

[3]. Semenyuk, N., Kostiv, U., Suberlyak, O., Skorokhoda, V. (2013). Peculiarities of Filled Porous Hydrogels Production and Properties. Chemistry and Chemical Technology, 7(1), 95-99. doi: 10.23939/chcht07.01.095.

[4]. Wei, L., Luo, S., McDonald, A. G., Agarwal, U. P., Hirth, K. C. et al. (2017). Preparation and Characterization of the Nanocomposites from Chemically Modified Nanocellulose and Poly(lactic acid). Journal of Renewable. Materials, 5(5), 410-422. doi:10.7569/JRM.2017.634144.

[5]. Skorokhoda, V., Semenyuk, N., Dziaman, I., Suberlyak, O. (2016). Mineral Filled Porous

Composites Based on Polyvinylpyrrolidone Copolymers with Bactericidal Properties. Chemistry and Chemical Technology, 10(2), 187-192. doi: 10.23939/chcht10.02.187.

[6]. Suberlyak, O. Skorokhoda, V., Kozlova, N., Melnyk, Yu., Semenyuk, N., Chopyk, N. (2014). The Polyvinylpyrrolidone Graft Copolymers and Soft Contact Lenses on Their Basis. Science Rise, 5(3/5), 52-57. doi: 10.15587/2313-8416.2014.33235.

[7]. Skorokhoda, V., Dziaman, I., Dudok, G., Skorokhoda, T., Bratychak, M., \& Semenyuk, N. (2019). The Ultrasonic Effect on Obtaining and Properties of Osteoplastic Porous Composites. Chemistry and Chemical Technology, 13(4), 429-435. doi: 10.23939/chcht13.04.429. 